\title{
Screening for nasal carriage Staphylococcus aureus and their antibiotic susceptibility pattern among the health care workers in a tertiary care hospital, Nepal
}

\section{Jha B', Sapkota J2, Sharma M³, Mishra B4, Bhatt CP5}

${ }^{1}$ Beena Jha, Lecturer; ${ }^{2}$ Jyotshna Sapkota, Assistant Professor; ${ }^{3}$ Manisha Sharma, Assistant Professor; ${ }^{4}$ Bhawesh Mishra, Lecturer, ${ }^{5}$ Chandra Prakash Bhatt, Professor; Department of Microbiology, Kathmandu Medical College Teaching Hospital, Kathmandu, Nepal

\begin{abstract}
Background: The presence of Staphylococcus aureus in anterior nares of health care workers may serve as a major source of infection in hospital environment and act as a reservoir for the spread of Methicillin Resistant $S$. aureus between patients. Objective: The present study was conducted to determine the frequency of nasal carriage of $S$. aureus among the health care workers of Kathmandu Medical College Teaching Hospital and to assess the antibioticsusceptibility patterns of the Methicillin Resistant S. aureus isolates.

Methodology: A total of 200 health care workers involved in the management of patients from the intensive care unit, Operation Theatre, postoperative wards, different wards and emergency department were screened for $S$. aureus by collecting their nasal swabs.

Results: Out of 200 health care workers, only 10(5\%) were S. aureus nasal carriers. Out of 10 S. aureus strains isolated six $(60 \%)$ were methicillin sensitive $S$. aureuswhereas four (40\%) were methicillin resistant $S$. aureus. The isolates were $100 \%$ sensitive to Linezolid, Vancomycin and Amikacin but showed highest resistant to Azithromycin (100\%), followed by Cefixime (60\%).

Conclusion: This study concluded that $5 \%$ of health care workers were S. aureus carriers and proportion of Methicillin Resistant S. aureus and Methicillin sensitive S. aureus was $60 \%$ and $40 \%$ respectively.
\end{abstract}

Key words: Cefoxitin; HCWs; MRSA; MSSA; Staphylococcus aureus.

\section{INTRODUCTION}

T he presence of S. aureus in anterior nares of health care workers (HCWs) may serve as a major source of infection in hospital environment and act as reservoir for the spread of methicillin resistant S. aureus (MRSA) between patients ${ }^{1}$. The screening of the nasal carriage in HCWs plays a very important role in infection control of MRSA in any health care setting. The identification of colonized staff members and their proper management prevents the spread of MRSA strains among the patients as well as other hospital staffs ${ }^{2}$. In Nepal several reports

Address for correspondence

Dr. Beena Jha

Lecturer

Department of Microbiology

Kathmandu Medical College, Duwakot, Bhaktapur, Nepal

E-mail: drbeenajha@gmail.com have been published with the MRSA prevalence rate of nasal carriage among HCWs ranging from $20.37-43.80 \%{ }^{3}$.

Infections caused by MRSA strains, which are most often multidrug resistant are increasing and therapy has become problematic ${ }^{4}$. The horizontal transfer of mec $A$ gene is the major mechanism responsible for conferring resistance to Methicillin and other betalactam antibiotics 5 . Active surveillance for patients colonized with MRSA is recommended to prevent MRSA infections in health care setting ${ }^{3,6}$. Identification of HCWs colonized with MRSA, combined with other precaution and taking care of hand hygiene will be helpful in reducing the transmission and controlling the spread of infection.

\section{METHODOLOGY}

Institutional ethical clearance was obtained from Institutional Review Committee before this study 
was conducted. A total of $200 \mathrm{HCWs}$ involved in the management of patients from the intensive care unit, Operation Theatre (OT), postoperative wards, orthopaedic, obstetrics and gynaecology, paediatrics, medicine, surgical wards and emergency department were screened for MRSA by collecting their nasal swabs. Sterile swab stick was pre-moistened with sterile normal saline and specimens were collected from the anterior nares of both the nostrils of HCWs. The swabs were inserted in the tube labelled and were transported at room temperature to the Microbiology laboratory of Kathmandu Medical College Teaching Hospital (KMCTH) Sinamangal without any delay in processing of sample for culture and sensitivity.

The nasal swabs thus obtained were inoculated on 10\% blood agar, MacConkey's agar and Mannitol salt agar medium. The plates were incubated aerobically at $35^{\circ} \mathrm{C}$ in ambient air for 24 hours and were examined for growth. S. aureus was identified by standard methods. Colonies suggestive of $S$. aureus showing golden yellow colonies on mannitol salt agar were identified using Gram stain, catalase test, slide and tube coagulase tests ${ }^{7}$. The isolates were confirmed as MRSA by lawn culture in Muller

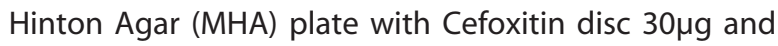
incubated at $35^{\circ} \mathrm{C}$ for 24 hours. Result was interpreted according to the Clinical Laboratory Standards Institute Guidelines (CLSI) $2013^{8}$. Zone of inhibition of $\geq 22$ were considered Methicillin sensitive and zone of inhibition $\leq$ 21 were considered methicillin resistant. Antimicrobial susceptibility testing were performed using modified
Kirby Bauer disc diffusion method on MHA; zone size were measured and interpreted as sensitive, intermediate and resistant. Antibiotic discs used were from HiMedia: Cefoxitin $(30 \mu \mathrm{g})$, Ciprofloxacin $(5 \mu \mathrm{g})$, Gentamycin $(10 \mu \mathrm{g}))$, Linezolid $(30 \mu \mathrm{g})$, Azithromycin $(30 \mu \mathrm{g})$, Cefixime $(30 \mu \mathrm{g}))$, and Vancomycin $(30 \mathrm{~g})$ and

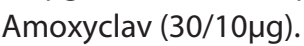

\section{RESULTS}

This study revealed that $10(5 \%)$ of $200 \mathrm{HCWs}$ are carriers of $S$. aureus in their anterior nares among which six (2\%) were methicillin resistant.

Nasal swabs obtained from $200 \mathrm{HCW}$ were processed for bacteriological culture and sensitivity of which $85(42.5 \%)$ were male and $115(57.5 \%)$ female. Most of the HCWs were among the age group 20-40 years. Out of 200 nasal swab samples, S. aureus were isolated from $10 \mathrm{HCWs}$. MRSA isolates of total nasal swab was four (2\%). Out of $10 \mathrm{~S}$. aureus strains isolated $6(60 \%)$ were methicillin sensitive S. aureus (MSSA) whereas four (40\%) were methicillin resistant $S$. aureus (MRSA).

The MRSA isolates were sensitive to Linezolid, Vancomycin and Amikacin, resistant to Azithromycin, followed by Cefixime. Reports were dispatched to the respective HCWs who were nasal colonizers of $S$. aureus and advised to apply Mupirocin topically in both the nostrils twice a day for seven days. Those HCWs were asked to give their repeat nasal swab samples to look for any resistance towards Mupirocin.

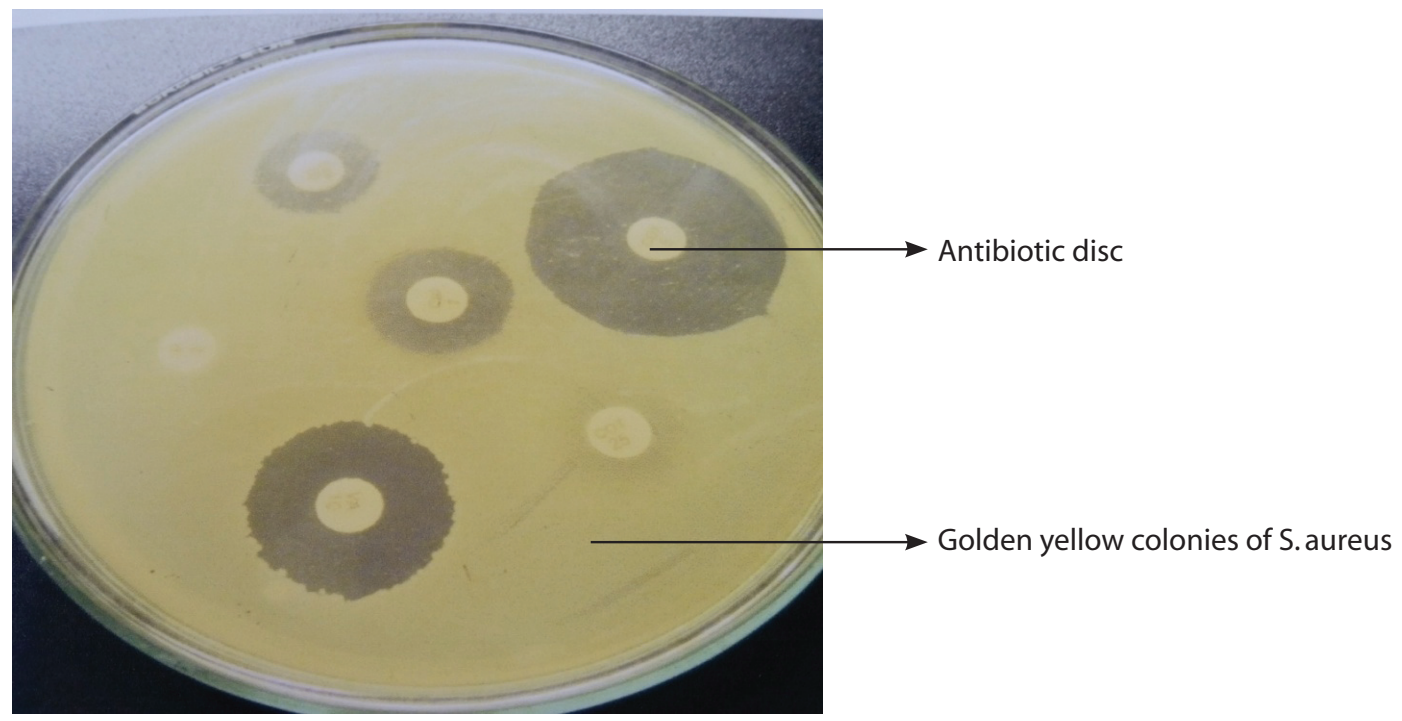

Figure 1: Antibiotic susceptibility pattern of S.aureus on MHA plate 
Table 1: Antibiotic susceptibility pattern in isolates from S. aureus sample

\begin{tabular}{lccc} 
Antibiotics & Sensitive & Intermediate & Resistant \\
\hline Gentamycin & $10(100 \%)$ & $0(0 \%)$ & $0(0 \%)$ \\
Ciprofloxacin & $5(50 \%)$ & $3(30 \%)$ & $2(20 \%)$ \\
\hline Azithromycin & $0(0 \%)$ & $0(0 \%)$ & $10(100 \%)$ \\
Linezolid & $10(100 \%)$ & $0(0 \%)$ & $0(0 \%)$ \\
Cefixime & $4(40 \%)$ & $0(0 \%)$ & $6(60 \%)$ \\
Vancomycin & $10(100 \%)$ & $0(0 \%)$ & $0(0 \%)$ \\
\hline Amoxyclav & $5(50 \%)$ & $1(10 \%)$ & $4(40 \%)$ \\
\hline
\end{tabular}

\section{DISCUSSION}

Nasal carriage rate of MRSA have been reported in a range of $6 \%$ to $17.8 \%$ among the health care workers in hospital settings elsewhere in the world ${ }^{9}$. In a similar study done in India by V. Rutvi et al. and Y. Sharma showed nasal carriage rate of MRSA to be $6 \%$ and $5 \%$ respectively, whereas the study done in Australia by Munckhof et al. showed only $0.7 \% \%^{10-12}$. In contrast to this study, a higher propensity was observed amongst MRSA strains reported in a study done by A. Currie et al. USA $(24.15 \%)^{13}$. All the MRSA isolates were sensitive to Linezolid, Vancomycin and Amikacin and were resistant to Azithromycin followed by Cefixime. These findings are consistent with a study done by Chatterjee et al. in which resistance to Ciprofloxacin being $12.5 \%$ but in sharp contrast to the results by Goyal R. et al where $60 \%$

\section{REFERENCES}

1. Kumar P, Shukla I, Varshney S. Nasal screening of healthcare workers for nasal carriage of coagulase positive MRSA and prevalence of nasal colonization with Staphylococcus aureus. Res Artic Biol Med [Internet]. 2011 [cited 2018 Feb 30];3(2):182-6. [ Full Text ]

2. Shinde RV, Pawar SK, Mohite Rv, Shinde AR, Duggu P. Study of Nasal Carriage of Staphylococcus aureus with Special Reference to Methicillin Resistance among Nursing Staff. Arch Clin Microbiol [Internet]. [cited 2018 Feb 30];7(1).[ Full Text ]

3. Khanal R, Sah P, Lamichhane $P$, Lamsal A, Upadhaya S, Pahwa VK. Nasal carriage of methicillin resistant Staphylococcus aureus among health care workers at a tertiary care hospital in Western Nepal. Antimicrob Resist Infect Control [Internet]. 2015 [cited 2018 Feb 30];4:39. [ PubMed ]

4. Voss A, Doebbeling BN. The worldwide prevalence of methicillin-resistant Staphylococcus aureus. Int J Antimicrob Agents [Internet]. 1995 Apr [cited 2018 Feb 30];5(2):101-6. [ PubMed ]
MRSA were resistant to Ciprofloxacin ${ }^{14,15}$. Both the above studies showed $100 \%$ sensitivity towards Vancomycin which is in accordance to our study. None of the isolates were multidrug resistant in the present study unlike Goyal et al. who reported 3/10 (30\%)-resistance to all the antibiotics tested $^{14}$.

Low MRSA nasal carriage prevalence could be related to active and periodic surveillance for MRSA nasal carriers among the HCWs at KMCTH. Periodic screening for MRSA nasal carriage among the HCWs and fumigation of operation theatre, intensive care units, post operative and other wards is conducted routinely. Pre and post fumigation culture of different wards are performed and report dispatched to the respective department which also helps to reduce the spread of MRSA strains circulating in the hospital environment.

\section{CONCLUSION}

This study concluded that $5 \%$ of HCWs were S. aureus carriers and proportion of MRSA and MSSA was $60 \%$ and $40 \%$ respectively. Regular surveillance of hospitalassociated infection and monitoring of antibiotic sensitivity pattern is required to reduce MRSA prevalence ${ }^{16}$. Screening and decolonization may be effective in decreasing the MRSA carriage rate among the HCWs. Standard infection control precautions should be employed to minimize either the carriage or the transmission rate.

5. Rybak MJ, LaPlante K. "Community-Associated MRSA: A Review,"Pharmacotherapy(Internet) 2005; 25(1):74-85. [ Full Text ]

6. Coia JE, Duckworth GJ, Edwards DI, Farrington M, Fry C, Humphreys $\mathrm{H}$, et al. Guidelines for the control and prevention of meticillin-resistant Staphylococcus aureus (MRSA) in healthcare facilities. J Hosp Infect [Internet]. 2006 May [cited 2018 May 30];63:S1-44. [PubMed]

7. Braid D. Staphylococcus: Cluster-forming Gram psitive cocci. In: Mackie and McCartney, Practical Medical MicrobiologyColle JG, Fraser AG, Marmiom BP, Simmons A, editors. Chapter $11.14^{\text {th }}$ ed. Churchill Livingstone INC;London:1996;244-58.

8. Clinical and Laboratory Standards Institute (CLSI). Performance standards for Antimicrobial susceptibility testing. Twenty- third informational supplement. M100-S23, Jan 2013.[cited 2018 Feb 30 ]:24-9. [ Full Text ]

9. Radhakrishna M, Monalisa D, Subbannayya K, SaralayaV, Kotian S. Prevalence of Methicillin Resistant Staphylococcus aureus Carriage amongst 
Health Care Workers of Critical Care Units in Kasturba Medical College Hospital, Mangalore, India. J Clin DIAGNOSTIC Res [Internet]. 2013 Dec [cited 2018 Feb 30];7(12):2697-700. [ PubMed ]

10. Vaidya R, Patel S, Bhatt S, Patel P. Nasal carriage rate of methicillin resistant Staphylococcus aureus (MRSA) among Civil Hospital health care workers. Int. J. Med. Public Health. 2016;6(4):180-83.[ Full Text ]

11. Sharma $Y$, Jain S, Singh H, Govil V. Staphylococcus aureus: Screening for Nasal Carriers in a Community Setting with Special Reference to MRSA. Scientifica (Cairo) [Internet]. 2014 [cited 2018 Feb 30];2014:15. [ PubMed]

12. Munckhof WJ, Nimmo GR, Schooneveldt JM, Schlebusch S, Stephens AJ, Williams G, et al. Nasal carriage of Staphylococcus aureus, including community-associated methicillin-resistant strains, in Queensland adults. Clin Microbiol Infect [Internet]. 2009 Feb [cited 2018 Feb 30];15(2):14955. [ PubMed]
13. Currie A, Davis L, Odrobina E, Waldman S, White $D$, Tomassi J, et al. Sensitivities of Nasal and Rectal Swabs for Detection of Methicillin-Resistant Staphylococcus aureus Colonization in an Active Surveillance Program. J Clin Microbiol [Internet]. 2008 Sep 1 [cited 2018 Feb 30];46(9):3101-3.[ PubMed ]

14. Chatterjee SS, Ray P, Aggarwal A, Das A, Sharma M. A community-based study on nasal carriage of Staphylococcus aureus. Indian J Med Res [Internet]. 2009 Dec [cited 2018 Feb 30];130(6):742-8. [ PubMed ]

15. Goyal R, Das S, Mathur M. Colonisation of methicillin resistant Staphylococcus aureus among health care workers in a tertiary care hospital of Delhi. Indian J Med Sci [Internet]. 2002 Jul [cited 2018 Feb 30];56(7):321-4. [ PubMed ]

16. Arora S, Devi P, Arora A, Arora U. Prevalence of methicillin-resistant Staphylococcus Aureus (MRSA) in a Tertiary Care Hospital in Northern India. J Lab Physicians [Internet]. 2010 Jul [cited 2018 Feb 30];2(2):78. [ PubMed ] 